\title{
1 Paper-based Sensing of Fucosylated Biological Compounds
}

2 Fatima Enam ${ }^{1}$, Emily Kramer ${ }^{1}$, Frederick Robinson ${ }^{2}$, Andrea Alvarez-Acosta $^{1}$, Rebecca

3 Cademartiri $^{3}$, Thomas J. Mansell ${ }^{* 1}$

4 1. Department of Chemical and Biological Engineering, lowa State University, Ames, IA, 50011

5 2. Department of Bioengineering, Rice University, Houston, TX, 77005

6 3. Department of Material Science and Engineering, lowa State University, Ames, IA, 50011

$7 \quad$ *Correspondence: $\underline{\text { mansell@iastate.edu }}$

\section{Summary}

Advances in sensing technology have enabled rapid analysis of various biomolecules including

11 complex carbohydrates. However, glycan analysis is limited by the throughput and complexity of

12 assays for quantifying them. We describe a simple, low-cost enzymatic assay for the rapid

13 analysis of fucosylation, down to linkage specificity, and its application to high-throughput

14 screening of biologically relevant fucosylated compounds, to facilitate simple and

15 straightforward analytical techniques. Paper-based devices integrate biosensor platforms and

16 other diagnostic assays by fusing them with wax printing technology, making their fabrication

17 even more inexpensive and simple. The specificity of the assay is established by linkage-

18 specific glycosidic enzymes and the colorimetric output is visible to the naked eye, with costs

19 that are lower than fluorescence/luminescence-based assays (\$0.02/reaction). This platform

20 was further improved by enhancing storage stability to retain analytical performance over time

21 using desiccation and freeze-drying techniques. The assay platform allows analysis of hundreds

22 of samples in minutes and we anticipate that this rapid and simple analytical method will be

23 extended towards developing a universal glyco-barcoding platform for high throughput

24 screening of glycosylation. 
26 Keywords: Fucose; paper-based device; high throughput sensing; human milk

27 oligosaccharides

\section{Introduction}

29 L-fucose is a common terminal sugar in glycans and oligosaccharides that has been attributed

30 to several key metabolic and structural functions ${ }^{1,2}$. It is found as a terminal modification on $\mathrm{N}$-,

31 O- or lipid-linked oligosaccharides and as a core modification on N-glycans or linked to serine

32 (Ser) or threonine (Thr) on proteins. It is also the second most abundant terminal

33 monosaccharide moiety, after sialic $\mathrm{acid}^{3}$, found in mammalian carbohydrates to form various

34 recognition motifs on many glycoproteins and glycolipids. Most notably, fucose has important

35 roles as a component of $\mathrm{ABO}$ blood group antigens ${ }^{4}$, a key modulator of interactions in the

36 immune system and in human breast milk.

37 Breastfeeding is associated with a lower risk of infection in babies and the underlying

38 mechanism has been linked to the diverse range of complex sugars ${ }^{5,6}$, the human milk

39 oligosaccharides (HMOs) ${ }^{7}$. A large majority of these HMOs are fucosylated ${ }^{8,9}$, with 2'-

40 fucosyllactose (2'-FL) being the most abundant HMO comprising approximately $2 \mathrm{~g} / \mathrm{L}$. Notably,

41 fucosylated HMOs inhibit binding and colonization of pathogens ${ }^{10,11}$ and provide protection

42 against diarrhea associated with stable toxin of Escherichia coli ${ }^{12,13}$. Supplementation of 2'-FL to

43 infant diet showed improved outcomes on innate and adaptive immune profiles ${ }^{14}$, and

44 increasing evidence of its health benefits has led to its commercialization as a supplement in

45 infant formula.

46 Similarly, the lipopolysaccharide (LPS) of Helicobacter pylori strains expresses Lewis

47 antigens ( $\mathrm{Le}^{\mathrm{x}}, \mathrm{Le}^{\mathrm{y}}, \mathrm{H}$ type I blood group structures) resembling the surface of host gastric

48 epithelial cells ${ }^{15}$ and upon $H$. pylori infection, this may cause antibodies to bind not only to the

49 bacteria but also the host epithelium resulting in tissue destruction. This antigen mimicry, being

50 analogous to $\mathrm{ABO}$ blood group antigens, may also provide persistence through immune 
51 evasion ${ }^{16}$. Fucosylation is also an important oligosaccharide modification involved in cancer and

52 inflammation ${ }^{17}$. The majority of human immunoglobulins $(\lg G)$ contain core fucosylation of the

$53 \mathrm{~N}$-glycan in the Fc region, the absence of which has shown to have enhanced antibody-

54 dependent cellular cytotoxicity (ADCC) activity due to increased binding affinity ${ }^{18,19}$.

55 Despite the biological importance of fucose, a critical obstacle in elucidating its

56 biochemical roles lies in the technical difficulties associated with detection and analysis,

57 especially with determining carbohydrate linkage positions. Current state-of-the-art methods for

58 determining fucosylation include capillary electrophoresis (CE), mass spectrometry (MS),

59 HPAEC-PAD ${ }^{20}, \mathrm{NMR}^{21}$ or glycan sequencing, which are laborious methods requiring

60 specialized equipment and sample preparation. Another established method with higher

61 throughput is the spectrophotometric detection of free L-fucose following defucosylation by

62 measuring the formation of $\mathrm{NADH}^{22}$. Seydametova et al. demonstrated quantitative assays for

632 '-FL, utilizing hydrolysis by fucosidases ${ }^{23,24}$. Their efforts, however, focused on a single

64 biological molecule and by spectrophotometrically measuring the end-point nicotinamide

65 adenine dinucleotide phosphate $\left(\mathrm{NADP}^{+}\right)$formed after an hour. Advances in synthetic biology

66 have enabled high-throughput screening via biological components that have been

67 standardized, for example, whole cell biosensors allow for the transduction of analyte

68 concentrations to changes in gene expression that facilitate high-throughput assaying ${ }^{25-27}$. We

69 previously demonstrated a genetically encoded whole cell biosensor to facilitate metabolic

70 engineering by enabling the linkage-specific high-throughput detection of 2'-FL and four other

$71 \mathrm{HMOs}^{28}$. The study demonstrated orthogonal screening of $\mathrm{HMO}$ structures, but entails culturing

72 bacterial strains relatively expensive equipment to read fluorescence, e.g., flow cytometer or

73 microplate reader.

74 To leverage the specificity and sensitivity of this assay to a faster and cheaper format,

75 we sought to transfer the underlying detection mechanism to a paper-based assay. Paper

76 based devices can be preferable to whole-cell assays, especially at the point of care, owing to 
77 their simplicity, low-cost and portability and the minimal resources, time and equipment required

78 in their fabrication ${ }^{29}$. These devices also allow for rapid analysis of multiple samples in parallel.

79 Colorimetric assays producing a visual readout are a common choice for paper-based

80 devices ${ }^{30-32}$, with their usefulness lying in the formation of brightly colored formazan products

81 upon reduction. Notably, they can be particularly useful in point-of-use or low-resource settings.

82 Here, we demonstrate a standard colorimetric assay for a simple, rapid, low-cost

83 linkage-specific sensing system for fucosylated biomolecules (Figure 1A). To create this device,

84 we expressed enzymes specific for three of the most common fucose linkages heterologously in

85 E. coli and immobilized them on to the paper device. Using a formazan reduction assay coupled

86 with fucose dehydrogenase, we then assay for the release of free L-fucose. The assay has a

87 limit of detection of below $20 \mathrm{mg} / \mathrm{L}$ for 2'-FL and can detect fucose linked to several types of

88 biomolecules, including HMOs, Lewis structures, and N-glycans. Finally, the assay can detect

89 fucosylated compounds in complex body fluid matrices such as human milk and simulated

90 urine.

91 Results

92 Fucosidases act as linkage-specific detection elements for fucosylated HMOs

93 To couple reduction of colorimetric dye to the quantification of 2'-FL, we used fucosidases

94 known in literature ${ }^{33,34}$ to be specific to the carbohydrate linkage to fucosylated molecules. Free

95 fucose can then be assayed using a redox-coupled reaction in which fucose dehydrogenase

96 (FDH) oxidizes L-fucose to fuconolactone, creating NADPH, which reduces the formazan dye.

97 To confirm that this coupled reaction scheme would produce NADPH, we incubated 2'-FL (Fuc-

$98 \alpha-1,2-$ Gal- $\beta-1,4-G l c)$ with commercial purified $\alpha-1,2$-fucosidase to cleave the fucose and in the

99 presence of commercial FDH and $\mathrm{NADP}^{+}$. By following the increase in absorbance at $340 \mathrm{~nm}$

100 due to reduction of $\mathrm{NADP}^{+}$on a microplate reader, we verified the redox reaction undergone by

101 the cleaved fucose (Supplementary Figure S1A). 
However, the use of two purified enzymes can be expensive. To achieve an efficient, low-cost detection system, we hypothesized that using a bacterial host as a heterologous system for the production of high levels of functionally active recombinant enzymes would be cost-effective. Because it efficiently and orthogonally cleaved L-fucose from 2'-FL in the context of a whole-cell biosensor ${ }^{35}$ we chose to express the soluble glycosyl hydrolase domain of AfcA, an $a-1,2-$ fucosidase from Bifidobacterium bifidum ${ }^{36}$ under a constitutive promoter, to liberate

108 fucose from 2'-FL ${ }^{35}$. Cell lysate from overnight cultures of E. coli BL21 (DE3) expressing AfcA 109 was added to purified FDH with $\mathrm{NADP}^{+}$as before. We detected reduction to NADPH when 2'-FL 110 was added as an analyte but not on the isomeric HMO 3-FL (Galß-1,4(Fuc-a-3)Glc)

111 (Supplementary Figure S1A). No background redox activity associated with the components in

112 the cell lysate itself was observed in an empty vector control over a period of one hour. This

113 strain served as a control in subsequent experiments, where the background noise from the 114 crude cell lysate was noted for all measurements.

Tetrazolium dye allows colorimetric detection of fucosylated compounds

117 Once we confirmed that the sequential reactions of cleavage by fucosidase followed by

118 oxidation by fucose dehydrogenase generated the reducing co-factor, we added a redox-

119 sensitive dye to make the assay visible by the naked eye. Tetrazolium salts have been

120 historically used for testing cell viability, proliferation and cytotoxicity ${ }^{37,38}$. We chose 2-(4-

121 iodophenyl)-3-(4-nitrophenyl)-5-phenyl-2H- tetrazolium chloride (INT), which in the presence of

122 the intermediate electron carrier, 5-methyl- phenazine methyl sulfate (PMS), is reduced to an

123 insoluble formazan. This precipitate absorbs light at a wavelength of $500 \mathrm{~nm}$ and provides a

124 robust signal visible to the naked eye ${ }^{39}$. The generated NADPH, when coupled to INT, forms a

125 stable red formazan (Figure 1B) and, being stoichiometrically proportional to the fucose

126 concentration allows us to directly quantify the fucose. We determined the optimum $\mathrm{pH}$ and 
127 buffer conditions for the enzyme cocktail in the assay and coupled it with the INT/PMS assay to 128 establish a colorimetric assay protocol.

129 We first sought to quantify free fucose with FDH from cell lysate. An intense color change was 130 observed with $500 \mathrm{mg} / \mathrm{L}$ L-fucose resulting in a $100 \%$ increase in absorbance within a few 131 seconds as read on a plate reader at $500 \mathrm{~nm}$ (Figure 1C). The reaction contains $2.5 \mu \mathrm{g}$ of L-

132 fucose with $\mathrm{NADP}^{+}$present in excess. In contrast, by measuring the NADPH formation by 133 reading the absorbance at $340 \mathrm{~nm}$, a $30 \%$ increase in absorbance was seen at equivalent L-

134 fucose concentrations, which is to be expected since the molar extinction coefficient of INT 135 formazan is $18000 \mathrm{M}^{-1} \mathrm{~cm}^{-1}$ compared to $6220 \mathrm{M}^{-1} \mathrm{~cm}^{-1}$ for NADPH. Thus, the colorimetric 136 system showed greater sensitivity than changes in NADPH readout as demonstrated by the 137 slopes in Supplementary Figure S3A. Notably, minimal background was again observed with 138 the empty vector control. In addition, the switch to a colorimetric reaction allowed us to scale 139 down the reaction volume to $20 \mu \mathrm{L}$.

140 To demonstrate the utility of the assay in reliably detecting fucosylated molecules, we used the 141 assay to detect 2'-FL using additional crude cell lysate from cells expressing pAfcA. We titrated 142 the amount of cell lysate needed for optimum signal. For each $20 \mu \mathrm{L}$ reaction, $5 \mu \mathrm{L}$ lysate was 143 added. This also exhibited strong absorbance, a four-fold increase, with 2'-FL (300 mg/L) at the 144 same concentration of L-fucose (300 mg/L), containing, as previously tested (Figure 1D). The 145 linkage specificity was validated by the lack of significant increase in absorbance when tested 146 with its isomer 3-FL, indicating that the $\alpha-1,3$ linkage was not defucosylated. Similarly, we went 147 on to demonstrate that this method can be extended to the detection of $\alpha-1,3$ linked fucose as 148 well by using lysate of cells harboring plasmid pAfcB expressing $\alpha-1,3-$ fucosidase under 149 identical experimental conditions. We observed a 1.4-fold change in absorbance with 3-FL but 150 no color change was observed with 2'-FL (Supplementary Figure S1B). Similar to our previous 151 biosensors, this assay also demonstrated linkage specificity and the ability to distinguish 152 between isomeric HMOs. 


\section{Fabrication of paper-based platform}

155 Once the colorimetric assay was developed, we focused on translating it to a paper-based

156 assay. Paper-based devices are fabricated from cellulose because it has advantages of being

157 low-cost, abundant, lightweight, biodegradable and biologically compatible ${ }^{40,41}$. In addition, its

158 porosity and hydrophilicity offers wicking properties due to capillary action that can handle small

159 volumes of liquid with no external pumping ${ }^{42}$. To create our device, we used Whatman Grade 3

160 chromatography paper and defined the hydrophilic area using wax-printing method owing to its

161 ease of printing using commercially available printers. The design layout for the paper

162 corresponded to the dimensions of a standard 96 -well plate ${ }^{43}$. We chose this to accommodate

163 distinct test zones for independent reactions that can be carried out in parallel, also allowing for

164 the use of multi-channel pipettors for rapid assaying. Once printed, we melted the wax in an

165 oven. To ensure fully formed barriers, we observed the bottom side of the paper. Prior to

166 heating, no pattern is visible at the back. Upon heating, the wax melts and vertically penetrates

167 through the porous fiber network ${ }^{44}$, which becomes visible on the bottom. This allows for the

168 aqueous sample and reagents to be contained within spatially separated hydrophilic zones.

169 There is also some lateral diffusion of the ink which reduces the diameter of the detection zone,

170 but this was taken into account during the design to ensure appropriate dimensions. We

171 designed the template with each well having a line thickness of $1 \mathrm{~mm}$ and internal diameter of 5

$172 \mathrm{~mm}$; after heating, the diameter was reduced to $4 \mathrm{~mm}$. Once cooled, we further tested the

173 hydrophobic barriers for leaks by addition of colored water and looking for any sort of bleeding

174 outside of the well. If the liquid failed to contain, the paper was heated longer until no bleed-

175 through was visible. The procedure is illustrated in Figure 2 and detailed in the Methods. Once

176 the device was ready to use, the enzymes were directly applied to the detection zones. A

177 master mix containing $\mathrm{NADP}^{+}$dissolved in Tris-HCl buffer $(\mathrm{pH} 9.5)$ was then added to wet the

178 test zones, followed by the analyte to be tested. Immediately following that, the dye was applied 
and allowed to dry under ambient conditions before scanning and analysis (Supplementary

Figure S2). In our experiments, it took less than $1 \mathrm{~min}$ from the addition of the dye for a detectable color change in the test zones to occur (Supplementary Video S1).

\section{Paper-based sensing for distinguishing fucosylated HMOs}

184 Next, we implemented this paper-based colorimetric assay for distinguishing three fucosylated

185 HMOS: 2'-FL (Fuc- $\alpha-1,2-G a l-\beta-1,4-G l c), 3-F L ~(G a l \beta-1,4(F u c-\alpha-3) G l c)$ and DFL (Fuc- $\alpha-1,2-G a l \beta-$

186 1,4(Fuc-a-3)Glc). We also tested for GDP-L-fucose, the donor substrate, a key component in

187 the biosynthesis of fucosylated $\mathrm{HMOs}^{45,46}$. Either synthetic routes for 2'-FL and 3-FL,

188 enzymatic $^{47}$ or microbial via metabolic engineering ${ }^{45}$, take place via GDP-L-fucose which can be

189 present in the final product at different levels. It is imperative that our assay is not sensitive to

190 GDP-L-fucose for its use in analysis of carbohydrate production from microbial sources. For the

191 assay we spotted the required enzymes on to the paper: either no enzyme as control and for

192 background reduction, only FDH for L-fucose and GDP-fucose detection, FDH and AfcA for 2'-

193 FL detection, FDH and AfcB for 3-FL detection and combination of FDH, AfcA and AfcB for DFL

194 detection. This was followed by the $\mathrm{NADP}^{+}$dissolved in the buffer, the respective analytes and

195 the dye. The paper was allowed to dry and for the color to fully develop for an hour at room

196 temperature before analysis. We first tested if the two isomers, 2'-FL and 3-FL were

197 distinguishable and if there was any crosstalk. We had established earlier the lack of substrate

198 promiscuity in AfcA and AfcB in the context of the whole cell biosensor ${ }^{28}$. No fucose was

199 liberated from 3-FL when AfcA was present (Figure 3A). Figure 3B shows a 25-reaction array

200 to distinguish three fucosylated HMOs. The control zones (top row with no added enzymes and

201 left column with no added substrate) showed no color change and the values obtained were

202 noted to account for the background signal from the dye and improve the accuracy of the assay.

203 Notably, no residual activity was observed with the concentrations of cell lysate used. An

204 intense color change from yellow to red was observed with free L-fucose when FDH was 
present, as expected. This served as a positive control, confirming the activity of the FDH. With 2'-FL, a color change was observed only when AfcA was present to cleave the $a-1,2$-linked

207 fucose. We thus obtained a signal only when AfcA or both AfcA and AfcB were present. We 208 obtained similar results with 3-FL when AfcB was present. We did not observe any color change 209 with GDP-fucose implying the lack of enzymatic activity of FDH on GDP-fucose as a substrate 210 (data not shown).

We generated the respective calibration curves by the addition of a series of dilutions of

212 2'-FL and 3-FL in range 0-6000 $\mathrm{mg} / \mathrm{L}$. We then scanned the paper device using a desktop

213 scanner and analyzed the digital image on the green intensity channel. We quantified the color

214 change in the test zones, and converted the color change value into the corresponding

215 concentrations using the calibration curve for the assay (Figure 3C, 3D). The experimental data

216 were fitted into a 4-parameter logistic model (4PL) using MATLAB and the resulting transfer

217 functions revealed the dynamic range and limits of detection. The calibration curve for 2'-FL

218 showed a wide dynamic range between 150 and $2500 \mathrm{mg} / \mathrm{L}$ of 2 '-FL $(0.3-5 \mathrm{mM})$ and the limit of

219 detection (LoD) was found to be below $20 \mathrm{mg} / \mathrm{L}(0.04 \mathrm{mM})$ which satisfies the levels at which

220 these biological molecules are produced either naturally or via synthesis. For example,

221 Baumgärtner et al. engineered an E. coli strain to produce 2'-FL at levels of up to $21 \mathrm{~g} / \mathrm{L}^{45}$ and

222 analysis of HMOs in human milk shows 2'-FL to be present $60-15000 \mathrm{mg} / \mathrm{L}^{48,49}$, which is well

223 within the limit of our device. Although the limit of detection is five-fold higher than our whole-cell

224 biosensor, the dynamic range for this device is much wider. Similarly, we characterized the

225 assay for 3-FL (Figure 3D) and the dynamic range of detection was found to be between 250

226 and $900 \mathrm{mg} / \mathrm{L}$ and the limit of detection below $250 \mathrm{mg} / \mathrm{L}(0.5 \mathrm{mM})$.

We also measured the absorbance values of the series of 2'-FL concentrations in a 96-

228 well paper plate on a microplate reader to see the correlation with the values obtained from the

229 paper-based assay. Supplementary Figure S3B shows the direct correlation of the 
230 absorbance values as measured with a plate reader with the values from the paper-based

231 assay which correlate quite well.

\section{Stability of paper-based sensor}

234 Since the intrinsic instability of enzymes or proteins in general, makes it challenging for them to

235 be used for downstream applications, it was important to establish that our assay was functional

236 over a longer period of time. Different strategies have been developed to produce more stable

237 proteins and improve storage stability and shelf-life. The most frequently used method,

238 especially in the biopharmaceutical industry is drying ${ }^{50,51}$. We spotted the enzymes on the test

239 zones and either dried it at room temperature or by lyophilization. We then evaluated the

240 stability of the enzymes on the cellulose matrix by storage at room temperature or at $4{ }^{\circ} \mathrm{C}$. We

241 performed the assay for 2'-FL detection under the four different conditions over a period of 45

242 days and imaged the wells to confirm expression of the enzymes (Supplementary Figure

243 S3C). The desiccated enzymes maintained the similar activity over a period of one week, and

244 slowly started to decline. In 5 weeks, the activity dropped to $50 \%$ of the original, under both

245 storage conditions, and to $35 \%$ in 45 days. However, when lyophilized, $70 \%$ of its activity was

246 retained after 45 days. It was noticed that with refrigeration, the decline in activity was slower

247 compared to storage at room temperature. In solution, the enzyme master mix loses its activity

248 at room temperature in one day and in less than a week at $4{ }^{\circ} \mathrm{C}$. Overall, the data demonstrates

249 sufficient stability of the paper-based sensor, especially under lyophilization. The assay can be

250 stored at room temperature for shorter durations, avoiding the need for cold-chain. Beyond

251 portability, immobilization of the enzymes enables minimal sample handling and cost and easy

252 operation, without the need for specialized equipment and skills. 
255 There is good evidence showing positive roles played by HMOs in breast milk, especially

256 fucosylated HMOs, on infant health. The $\alpha-1,2$ fucosyltransferase (FUT2) gene catalyzes the

257 transfer of $\alpha-1,2$-linked fucose residue, while the $\alpha-1,3 / 4$ fucosyltransferase (FUT3) transfers $\alpha$ -

258 1,3-linked fucose residues to HMOs. Secretor status (harboring a functional copy of the FUT2

259 gene) can be established by profiling of HMOs in breast milk ${ }^{52}$. This variation in the composition

260 of HMOs in different mothers can provide some infants advantages over others. We tested if our

261 assay was capable of detecting and quantifying 2'-FL and 3-FL in human breast milk to help

262 identify secretors. As a control, we used powdered cow's milk, reconstituted to mimic breast

263 milk. We also chose to validate the quantitation using HPLC/MS-MS based on standard

264 retention times and mass spectrometric analysis. For the human breast milk samples, we

265 observed a color change indicating a concentration of 5,012 mg/L of 2'-FL (Figure 4A), which

266 we confirmed with a series of dilutions, and consistent with the 5-15 g/L of HMO typically

267 observed ${ }^{49}$. The signal from the paper-based assay indicating higher 2'-FL concentrations may

268 be attributed to the presence of other $\alpha-1,2$-fucosylated HMOs, such as lacto- $\mathrm{N}$-fucopentaoses

269 (LNFP I, II, III and V). The concentration of 3-FL was below the detection limit of the paper-

270 based assay. However, we observed a color change once the samples were concentrated five

271 and ten-fold (Figure 4B). 3-FL was found at $138 \mathrm{mg} / \mathrm{L}$ which corresponds to values found in

272 literature ${ }^{49}$. We observed no color change with the cow's milk samples, validating the

273 robustness of the assay in measuring fucosylation in biological fluids. To validate the assay,

274 characterization of isomers, 2'-FL and 3-FL, were carried out using mass spectrometry.

275 Although we were able to get adequate separation between the 2'-FL and 3-FL peaks, there

276 was overlap. The peaks were fully resolved by utilizing differences in $\mathrm{m} / \mathrm{z}$ ratios of the

277 deprotonated negative ions (MS) in conjunction with the difference in retention time (HPLC) to

278 resolve the isomers (Supplementary Figure S4). To confirm the identity of the peaks, we also

279 carried out an exoglycosidase ( $\alpha-1,2$-fucosidase or $\alpha-1,3$-fucosidase) digest. Treatment with

280 either $\alpha-1,2$-fucosidase or $\alpha-1,3$-fucosidase resulted in removal or major reduction of the 
expected peaks (Supplementary Figure S5). HMOs have also been detected in the serum and urine of mothers, with 2'-FL at the highest concentrations in secretors $(20 \mathrm{mg} / \mathrm{L})^{53}$. Studies have also shown high resemblance of HMO profiles in infant urine to mother's breast milk, as some are absorbed and reach the systemic circulation and excreted in urine ${ }^{54}$. These samples can serve as excellent indicators of availability of fucosylated HMOs to infants, allowing for supplementation when needed. We spiked non-biological urine containing constituents that mimic human urine with 2'-FL within a physiologically relevant concentration range. The levels of 2'-FL in urine have been shown to correspond to levels in the milk. Goehring et al. found the urine of breast-fed babies of secretor mothers to contain 2'-FL in the range of $35-191 \mathrm{mg} / \mathrm{L}^{55}$. The assay responded to both low (20 mg/L) and high (200 mg/L) levels of 2'-FL spiked in Surine (Figure 4C). Importantly, we did not see any interference from the urine constituents with the enzymes in our assay.

\section{Determination of fucosylation in glycoconjugates}

Glycoconjugates comprise a complex and diverse group of molecules that play important fucosylated glycoconjugates. We targeted the highly fucosylated blood group Lewis antigens, specifically the Type 2 structures: composed of Gal- $\beta(1,4)$-GlcNAc, giving rise to Lewis ${ }^{x}\left(\operatorname{Le}^{x}\right)$, sialyl-Lewis ${ }^{x}\left(\right.$ Sia-Le $\left.^{x}\right)$ and Lewisy $\left(\right.$ Le $\left.^{y}\right)$. We also tested the blood group $\mathrm{H}$ antigen, which is the fucosyl residue in blood group antigens. Due to the presence of either one or both of $\alpha-1,2 / 3 / 4-$

302 linked fucose, we took a combinatorial approach. With antigen $\mathrm{H}$ type 2, a colorimetric change 303 was only observed with AfcA (Figure 4D). Similarly, Le ${ }^{x}$ was only detected in the presence of 304 AfcB. With Le $e^{y}$ antigen, a three-fold increase in signal in the presence of two fucosidases was 305 observed. Sia-Le ${ }^{x}$ is sialylated and we hypothesized that the sialic acid may interfere with the 306 fucosidase activity. To test the hypothesis, we also introduced an a-2,3-neuraminidase that can 
cleave the sialic acid bound to the LacNAc. We observed a two-fold increase in the signal upon removal of sialic acid. Although the assay is not orthogonal, it allows us to easily distinguish the Lewis Type 2 antigens. This tool has the potential to determine the composition of HMOs in the

310 milk of mothers as determined by the Lewis blood group and hence, further aid in the

311 development of more personalized HMO supplementation. We next sought to determine if our

312 paper-based assay had the capacity to detect fucosylation in the context of more complex

313 biomolecules like glycoproteins. We targeted two common glycoproteins that are $\alpha-1,3-$

314 fucosylated: horseradish peroxidase (HRP) and phospholipase A2 (PLA2) to serve as models.

315 Core $a-1,3-F u c$ is a common N-glycan modification found frequently on many plant and insect

316 glycoproteins, but not mammalian ones. Since this core antigen does not occur in mammals it

317 may be responsible for inducing immunogenic responses in mammals ${ }^{56}$. These experiments

318 were consistent with our previous results; we were able to see a strong signal in the presence of

319 a-1,3 fucosidase with both glycoproteins (Figure 4E).

320 One common mammalian N-glycan modification is core $\alpha-1,6$ fucosylation. To specifically

321 cleave a-1,6 fucosylated N-glycans, we produced AlfA from Lactobacillus casei by recombinant

322 expression in E. coli as described earlier. AlfA is an $a-1,6$ fucosidase, shown to have specific

323 hydrolytic activity on $a-1,6$ linked fucose $e^{57}$. The recombinant enzyme was tested and validated

324 on 6'-fucosyl-GlcNAc and A1F-Glycan. 6'-Fuc-GlcNAc is an important modification of the core

325 glycan in N-glycoproteins. A1F-Glycan is a monosialo-fucosylated biantennary oligosaccharide,

326 found on different mammalian glycoproteins including IgG, gamma globulins, and many serum

327 glycoproteins. We observed a strong color change with both substrates and corresponded well

328 with the amount of fucose in the molecules (Figure 4F). The figures correspond to $0.4 \mu \mathrm{g}$ and 2

$329 \mu \mathrm{g}$ of L-fucose in A1F-Glycan and 6'-Fuc-GlcNAc respectively. We also tested for the specificity

330 of AlfA: no signal was seen with a-1,2/3 linked fucosylated substrates (2'-FL and 3-FL

331 respectively). This indicates that the paper assay allows for efficient, linkage specific

332 quantification of fucosylation in N-glycans. We attempted to detect $\alpha-1,6$ fucosylation in $\lg G$ 
333 from human serum but did not see any signal, possibly due to fucose being below the limit of

334 detection. No color change was observed when we tried analyzing the isolated N-glycans by

335 treatment of $\lg G$ with endoglycosidase PNGase A/F.

336

\section{Discussion}

338 As the role of carbohydrate structures in myriad biological processes is increasingly recognized,

339 rapid and low-overhead analysis of carbohydrates becomes increasingly important. For

340 example, fucosylation in serum alpha-fetoprotein can serve as a marker for early diagnosis of

341 hepatocellular carcinoma ${ }^{58}$. However, current methods of characterizing glycans, especially to

342 the level of linkage specificity, require expensive equipment and significant expertise. To enable

343 more practical methods of diagnosis or development of glycoprotein therapeutics, advances in

344 analytical glycomics are needed to overcome the bottlenecks associated with state-of-the art

345 technologies. Here we demonstrate a robust, inexpensive, high-throughput colorimetric assay

346 to quantify fucose in biologically relevant fucosylated molecules. The main advantage of this

347 assay is easy and quick way to perform it, enabling in-line detection of multiple samples in

348 parallel. This assay also minimizes the volume of sample needed for analysis. While this assay

349 can be useful in low-resource settings, it also has the potential to leverage the metabolic

350 engineering efforts for production of fucosylated glycans by enabling the high-throughput

351 screening of libraries of mutant variants. This can also provide a new approach for directed-

352 evolution efforts towards improved fucosyltranferase variants. In addition, integration of this

353 method with other glycosyl hydrolases beyond fucosidase could be leveraged to provide

354 comprehensive quantification of sugars and linkages in many complex carbohydrates. This

355 platform demonstrates high specificity, reproducibility and stability, which constitutes a

356 promising approach to be implemented as an easy-to-use protocol for sensitive and high-

357 throughput detection of fucose in biological samples. 


\section{Acknowledgements}

360 This work was supported by lowa State University Startup Funds. F.E. was funded by the NSF

361 Trinect Fellowship and Manley Hoppe Professorship. T.J.M. was partially supported by the

362 Karen and Denny Vaughn Faculty Fellowship. Frederick Robinson was supported by the NSF

363 BioMaP REU program. The authors thank Dr. Ludovico Cademartiri for assistance with wax-

364 printing. The authors also thank Dr. Lucas J. Showman at the W.M. Keck Metabolomics

365 Research Laboratory for helping to analyze the human breast milk samples.

366

367 Competing Interests Statement

368 The authors declare no competing interests.

\section{Online Methods}

\section{Chemicals and reagents}

372 All reagents used were of analytical grade. Tris- $\mathrm{HCl}$ buffer $(\mathrm{pH} 9.5)$ was used as the buffer

373 solution. 2-(p-iodophenyl)-3-(p-nitrophenyl)-5-phenyltetrazolium chloride (INT), phenazine

374 methosulfate (PMS), NADP ${ }^{+}$and dimethyl sulfoxide (DMSO) were purchased from Sigma-

375 Aldrich (Millipore Sigma, St. Louis, MO). Luria-Bertani (LB) culture medium, kanamycin and

376 carbenicillin were obtained from Sigma-Aldrich. Isopropyl b-D-1-thiogalactopyranoside (IPTG)

377 was purchased from Invitrogen (Invitrogen, Carlsbad, CA). 2'-Fucosyllactose and 3-

378 fucosyllactose were a kind donation by Glycom (Glycom A/S, Denmark). L-fucose, GDP-fucose,

379 A1F-Glycan and 2-Acetamido-2-deoxy-6-O-(6-deoxy-a-L-galactopyranosyl)-D-glucopyranose

380 (Fuc-a-1,6-GlcNAc) were purchased from Carbosynth (Carbosynth, Berkshire, UK).

381 Phospholipase A2 (Apis mellifera) was purchased from Enzo (Enzo Life Sciences Inc.,

382 Farmingdale, NY) and horseradish peroxidase from Sigma. L-Fucose Assay Kit was purchased 
from Megazyme. All assay reagents were stored at $-20^{\circ} \mathrm{C}$. The assays were carried out at room

384 temperature.

\section{Bacterial strains and plasmids}

387 E. coli NEB 5-alpha (New England Biolabs Inc., Ipswich, MA) was used for routine cloning and 388 E. coli BL21 (DE3) was used for protein overexpression. Primers and synthetic DNA were 389 purchased from Integrated DNA Technologies (IDT). Construction of plasmid pAfcA and pAfcB 390 is reported in our previous work ${ }^{28}$. Plasmids pAfcA and pAfcB comprises of a constitutive 391 promoter and the fucosidase gene cloned onto a G9m-2 vector. Plasmid pET28:FDH was 392 constructed by replacement of GFP in the pET28:GFP plasmid with the fucose dehydrogenase 393 (FDH) gene with a His-tag fusion at the N-terminal. The FDH gene was amplified by PCR from 394 the genomic DNA of Bifidobacterium longum subsp. infantis ATCC 15697TM (American Type

395 Culture Collection, Manassas, VA). The FDH fragment was assembled into the pET28b vector, 396 downstream of a T7 promoter, using NEBuilder® HiFi DNA Assembly Master Mix (New England 397 Biolabs Inc., Ipswich, MA). The AlfA gene from L. casei was constructed by DNA synthesis 398 (IDT) after codon-optimization for E. coli (IDT DNA Codon Optimization Tool). The gBlock was 399 amplified and assembled into the G9m-2 vector, downstream of the constitutive promoter.

400 Bacterial transformations were carried out using electroporation. The authenticity and 401 orientation of the inserts was confirmed by DNA sequencing at the lowa State DNA Facility 402 (Ames, IA).

\section{Proof of concept using commercial kit}

405 To test for the viability of using INT/PMS for a colorimetric readout for assaying free fucose, we 406 used a L-Fucose Assay Kit (Megazyme Inc., Chicago, IL). Different concentrations of L-fucose 407 were prepared and the assay carried out in a 96-well plate, according to the protocol supplied 408 by the manufacturer. $2 \mu \mathrm{L}$ of the dye mix was added to the reaction mixes and allowed to react 
for 5 mins. To test for the release of fucose from 2'-FL and 3-FL, a range of concentrations of

410 the oligosaccharides were incubated with lysate from cells expressing pAfcA and pAfcB

411 respectively, and followed by the kit protocol. The absorbance at $500 \mathrm{~nm}$ was monitored

412 throughout the reaction.

\section{Enzyme isolation}

415 The fucosidase and FDH expressing strains were cultured in $50 \mathrm{~mL}$ of LB medium at $37^{\circ} \mathrm{C}$ at

$416250 \mathrm{rpm}$, overnight with appropriate antibiotics (carbenicillin, $50 \mathrm{ug} / \mathrm{ml}$ and $30 \mathrm{ug} / \mathrm{ml} \mathrm{kanamycin}$,

417 respectively). The pET28:FDH was induced with IPTG $(0.5 \mathrm{mM})$ at mid-log phase. These

418 cultures were then incubated at $37^{\circ} \mathrm{C}$ in a rotary shaker $(250 \mathrm{rpm})$ for $16 \mathrm{hr}$. The overnight

419 cultures were washed and resuspended in $2 \mathrm{~mL}$ water, and lysed by sonication (10 min, $30 \mathrm{~s}$

420 pulse, $30 \mathrm{~s}$ off, $30 \%$ amplitude). Debris was removed from lysates by centrifugation at $5000 \mathrm{xg}$

421 for 30 mins and the supernatant stored at $4{ }^{\circ} \mathrm{C}$. One unit of a-1,2-L-fucosidase activity was

422 defined as the amount of enzyme required to release one mmol of L-fucose per minute from 2'-

$423 \mathrm{FL}(2 \mathrm{mM})$ in sodium phosphate buffer $(10 \mathrm{mM}), \mathrm{pH} 7$ at $30^{\circ} \mathrm{C}$. The $\mathrm{N}$-terminally His-tagged

424 FDH was purified from the lysate under native conditions using the QIAexpress Ni-NTA Fast

425 Start Kit (Qiagen, Vallencia, CA). $5 \mu$ of each fraction was mixed with $5 \mu$ l of 2x Laemmli

426 Sample Buffer (Bio-Rad, Hercules, CA). Each sample was heated at $95^{\circ} \mathrm{C}$ for 5 minutes and

427 resolved on SDS-PAGE. The gel was visualized by Coomassie brilliant blue staining. The

428 apparent molecular weight of FDH was approximately $32 \mathrm{kDa}$. The protein expression yields

429 were calculated using a NanoDrop One Spectrophotometer (Thermo Scientific, Waltham, MA).

\section{Fabrication of paper-based sensor}

432 The patterns for this study were printed on Whatman® grade 3 filter papers via wax-printing.

433 They were purchased as sheets measuring $460 \mathrm{~mm} \times 570 \mathrm{~mm}$ and cut to letter-size. Filter

434 paper (Whatman \#3) was chosen for its wicking properties and sturdiness, compared to filter 
papers of other thicknesses. The detection zone patterns were designed as circles with $7 \mathrm{~mm}$

436 inner diameter and $1 \mathrm{~mm}$ thickness on Adobe Illustrator (Adobe, San Jose, CA). The detection

437 zones were printed with a Xerox Color Qube 8570DN'TM (Xerox, Norwalk, CT) printer using

438 black hydrophobic wax-based ink.

439 The printed paper was placed in an oven set at $180^{\circ} \mathrm{C}$ for 5 mins. To assess if the hydrophobic

440 barriers fully penetrated the paper, water was spotted onto the detection zones to identify any

441 bleeding and cross contamination between zones. If the water bled through, the paper was

442 reheated and tested again till no bleed through was observed. The paper was used once cooled

443 to room temperature. A layer of single-sided tape was used to seal the bottom side of the paper

444 to contain the liquid within the barrier and prevent leakage.

\section{Performing paper-based assay}

447 The lysates containing the enzymes were spotted at the center of the detection zones and

448 allowed to dry. A $20 \mu \mathrm{L}$ reaction system contained $2.5 \mathrm{uM} \mathrm{NADP}^{+}, 1.25 \mathrm{mM}$ INT (in DMSO) and

$4490.5 \mathrm{mM}$ PMS (in DMSO). Table 1 shows a full listing of volumes of reagents required per

450 reaction. A mastermix containing all the reagents excluding the enzymes and the dye was

451 made and added to each well. The reactions were initiated by addition of the

452 samples/standards, immediately followed by the addition of the dye reagent. Reactions were set

453 up under low-light conditions. A color change was observed within 5 mins. All measurements

454 were performed in triplicate. The paper was left to incubate in the dark till dry. Once dry, the

455 paper was scanned using an Epson Perfection V800 Photo Scanner. To evaluate the limit of

456 detection and generate a standard curve, a series of dilutions of 2'-FL/3-FL were made and

457 analyzed. Data were fit via non-linear regression analysis using MATLAB.

458

459 Image and Statistical Analysis 
460 The scanned images were analyzed on ImageJ that provided intensity data for each reaction.

461 The MicroArray plugin was used that uses internally controlled regions of interest (ROI) and can

462 measure microarray image stacks. The RGB channels were split and intensities were analyzed

463 in the green channel and the mean values recorded. The background from the control with no

464 analyte was calculated for each measurement. Calibration curves (Figure 3C, 3D) were built to

465 get a quantitative readout from the measured transmittance. Comparison of our assay against a

466 commercial microplate-reader was also carried out. We defined the LoD as the lowest fucose

467 concentration that gave a colorimetric signal significantly above the control (no fucosylated

468 analyte) sample $(P \leq 0.01)$. $P$ values were determined by performing a paired parametric $t$ test

469 on Origin. To compare the analytical performance of our assay on biological fluids, we analyzed

4706 random trials, with one half of the experimental data set allocated toward blind testing.

\section{Table 1: Procedure for running assay}

\begin{tabular}{|l|l|l|l|}
\hline Reagent & Standard & Blank & Sample \\
\hline Distilled water & $10 \mu \mathrm{L}$ & $11 \mu \mathrm{L}$ & $10 \mu \mathrm{L}$ \\
\hline Buffer & $2 \mu \mathrm{L}$ & $2 \mu \mathrm{L}$ & $2 \mu \mathrm{L}$ \\
\hline NADP $^{+}$ & $0.5 \mu \mathrm{L}$ & $0.5 \mu \mathrm{L}$ & $0.5 \mu \mathrm{L}$ \\
\hline FDH & $0.1 \mu \mathrm{L}$ & $0.1 \mu \mathrm{L}$ & $0.1 \mu \mathrm{L}$ \\
\hline Fucosidase & $1 \mu \mathrm{L}$ & $1 \mu \mathrm{L}$ & $1 \mu \mathrm{L}$ \\
\hline
\end{tabular}




\begin{tabular}{|l|l|l|l|} 
Sample/standard & $5 \mu \mathrm{L}$ & - & $5 \mu \mathrm{L}$ \\
\hline INT/PMS dye & $2 \mu \mathrm{L}$ & $2 \mu \mathrm{L}$ & $2 \mu \mathrm{L}$ \\
\hline
\end{tabular}

\section{Long term stability of the paper-based assay}

475 To assess the stability of the paper assay, the AfcA enzyme lysates were spotted onto the 476 detection zones and left to air-dry in the fume hood for an hour at room temperature. Another 477 batch of spotted paper was subjected to lyophilization overnight. The papers were either stored 478 at room temperature or at $4{ }^{\circ} \mathrm{C}$ in plastic bags with silica pouches in the dark. To determine the 479 enzymatic activity after specific storage time, the assays for detection of 2'-FL were carried out 480 over a period of 45 days by rehydration with standard solutions. The colorimetric output was 481 analyzed as described earlier. All measurements were carried out at room temperature and in 482 triplicates.

\section{Analysis of biological samples}

485 Pooled, deidentified human breast milk samples were purchased from BioIVT (Westbury, NY). 486 Surine ${ }^{\mathrm{TM}}$ Negative Urine Control was purchased from Cerilliant (Cerilliant Co., Round Rock, 487 TX). HMOs were extracted from human breast milk using established procedures. $200 \mu \mathrm{L}$ of 488 milk was centrifuged at $9,000 \mathrm{rpm}$ for $20 \mathrm{mins}$ at $4{ }^{\circ} \mathrm{C}$ to remove lipids. After the top layer was 489 removed, $400 \mu \mathrm{L}$ of ethanol was added and centrifuged at $9,000 \mathrm{rpm}$ for $10 \mathrm{mins}$ at $4{ }^{\circ} \mathrm{C}$ and

490 the supernatant collected. The ethanol was removed by vacuum centrifuging and reconstituted 491 in water to different concentrations before analysis using the assay and validation of relative 492 abundance carried out using LC-MS/MS. We used an Agilent Technologies 1100 Series HPLC 493 system coupled to an Agilent Technologies Mass Selective Trap SL detector (Agilent 
494 Technologies, Santa Clara, CA) equipped with an electrospray ion source (ESI) and controlled

495 by ChemStation A.10.02, including data acquisition and processing. Both MS and MS/MS

496 spectra were acquired in the negative-ion mode with an acquisition rate of $1 \square$ s per spectrum

497 over ranges of $\mathrm{m} / \mathrm{z} 300$ to 2,000 (for MS) and m/z 50 to 2,000 (for MS/MS). Precursor-ion

498 selection was performed automatically by the data system based on ion abundance. Three

499 precursors were selected from each MS spectrum to carry out product-ion scanning. Products

500 were analyzed on a Prevail Carbohydrate ES $(250 \mathrm{~mm} \times 4.6 \mathrm{~mm}$ id; $5 \mu \mathrm{m}$; Alltech Associates,

501 Deerfield, IL). The mobile phase consisted of water (aqueous solution containing $5 \mathrm{mM}$

502 ammonium acetate) and acetonitrile (AcN containing $5 \mathrm{mM}$ ammonium acetate). The solvent

503 gradient was delivered at a flow rate of $1 \square \mathrm{ml} / \mathrm{min}$ and consisted of an initial linear increase from

$50485 \%$ to $50 \%$ AcN over $10 \mathrm{~min}$, held at $50 \%$ AcN for 2 mins and followed by a linear decrease to

505 starting conditions for $2 \mathrm{~min}$. The injection volume was $5 \square \mu$ l. The dynamic range and linearity

506 for the quantification of 2'-FL and 3-FL were evaluated through the use of a series of dilutions as

507 standard curves. To analyze 3-FL, the samples were concentrated in a vacuum centrifuge

508 (Eppendorf, Hauppauge, NY). Disparities with other cited literature may be due to different

509 analytical technique and differences in biochemical extraction process.

\section{References}

511 1. Dalziel, M., Crispin, M., Scanlan, C. N., Zitzmann, N. \& Dwek, R. A. Emerging principles for 512 the therapeutic exploitation of glycosylation. Science 343, 1235681 (2014).

513 2. Schneider, M., Al-Shareffi, E. \& Haltiwanger, R. S. Biological functions of fucose in

514 mammals. Glycobiology 27, 601-618 (2017).

515 3. Werz, D. B. et al. Exploring the structural diversity of mammalian carbohydrates

516 ('glycospace') by statistical databank analysis. ACS Chem. Biol. 2, 685-691 (2007).

517 4. Lowe, J. B. The blood group-specific human glycosyltransferases. Baillieres. Clin.

$518 \quad$ Haematol. 6, 465-492 (1993). 
519 5. Ninonuevo, M. R. et al. A strategy for annotating the human milk glycome. J. Agric. Food $520 \quad$ Chem. 54, 7471-7480 (2006).

521 6. Stahl, B. et al. Oligosaccharides from Human Milk as Revealed by Matrix-Assisted Laser 522 Desorption/lonization Mass Spectrometry. Anal. Biochem. 223, 218-226 (1994).

523 7. Triantis, V., Bode, L. \& van Neerven, R. J. J. Immunological Effects of Human Milk

524 Oligosaccharides. Front Pediatr 6, 190 (2018).

525 8. Newburg, D. S. Oligosaccharides in human milk and bacterial colonization. J. Pediatr.

526 Gastroenterol. Nutr. 30 Suppl 2, S8-17 (2000).

527 9. Bode, L. Human milk oligosaccharides: every baby needs a sugar mama. Glycobiology 22, $528 \quad 1147-1162(2012)$.

529 10. Yu, Z.-T. et al. The principal fucosylated oligosaccharides of human milk exhibit prebiotic 530 properties on cultured infant microbiota. Glycobiology 23, 169 (2013).

531 11. Chichlowski, M., German, J. B., Lebrilla, C. B. \& Mills, D. A. The influence of milk 532 oligosaccharides on microbiota of infants: opportunities for formulas. Annu. Rev. Food Sci. 533 Technol. 2, 331-351 (2011).

534 12. Morrow, A. L., Ruiz-Palacios, G. M., Jiang, X. \& Newburg, D. S. Human-milk glycans that inhibit pathogen binding protect breast-feeding infants against infectious diarrhea. J. Nutr.

13. Newburg, D. S. et al. Innate protection conferred by fucosylated oligosaccharides of human

14. Goehring, K. C. et al. Similar to Those Who Are Breastfed, Infants Fed a Formula Containing 2'-Fucosyllactose Have Lower Inflammatory Cytokines in a Randomized Controlled Trial. J. Nutr. 146, 2559-2566 (2016). lipopolysaccharide and host Lewis blood group antigens in autoimmunity. Infect. Immun. 64, 2031-2040 (1996). 
16. Appelmelk, B. J. \& Vandenbroucke-Grauls, C. M. H pylori and Lewis antigens. Gut 47, 1011 (2000).

17. Miyoshi, E. et al. Fucosylation is a promising target for cancer diagnosis and therapy. Biomolecules 2, 34-45 (2012).

18. Satoh, M., lida, S. \& Shitara, K. Non-fucosylated therapeutic antibodies as next-generation therapeutic antibodies. Expert Opin. Biol. Ther. 6, 1161-1173 (2006).

19. Raju, T. S. Terminal sugars of Fc glycans influence antibody effector functions of IgGs. Curr. Opin. Immunol. 20, 471-478 (2008).

20. Kandzia, S. \& Costa, J. N-glycosylation analysis by HPAEC-PAD and mass spectrometry. Methods Mol. Biol. 1049, 301-312 (2013).

21. Grass, J. et al. Discovery and structural characterization of fucosylated oligomannosidic Nglycans in mushrooms. J. Biol. Chem. 286, 5977-5984 (2011).

22. Tsay, G. C. \& Dawson, G. A sensitive spectrophotometric method for detection of I-fucose. Anal. Biochem. 78, 423-427 (1977).

23. Seydametova, E. et al. Development of a quantitative assay for 2 '-fucosyllactose via onepot reaction with a1,2-fucosidase and l-fucose dehydrogenase. Anal. Biochem. 582,

24. Seydametova, E., Shin, J., Yu, J. \& Kweon, D.-H. A Simple Enzymatic Method for Quantitation of 2'-Fucosyllactose. J. Microbiol. Biotechnol. 28, 1141-1146 (2018).

25. van der Meer, J. R. \& Belkin, S. Where microbiology meets microengineering: design and applications of reporter bacteria. Nat. Rev. Microbiol. 8, 511-522 (2010).

26. Harms, H., Wells, M. C. \& van der Meer, J. R. Whole-cell living biosensors--are they ready for environmental application? Appl. Microbiol. Biotechnol. 70, 273-280 (2006). Biological Recognition with Reporter Genes. Chem. Rev. 100, 2705-2738 (2000).

28. Enam, F. \& Mansell, T. J. Linkage-Specific Detection and Metabolism of Human Milk 
Oligosaccharides in Escherichia coli. Cell Chem Biol 25, 1292-1303.e4 (2018).

29. Martinez, A. W., Phillips, S. T., Whitesides, G. M. \& Carrilho, E. Diagnostics for the developing world: microfluidic paper-based analytical devices. Anal. Chem. 82, 3-10 (2010).

30. Vella, S. J. et al. Measuring markers of liver function using a micropatterned paper device designed for blood from a fingerstick. Anal. Chem. 84, 2883-2891 (2012).

31. Hice, S. A., Santoscoy, M. C., Soupir, M. L. \& Cademartiri, R. Distinguishing between metabolically active and dormant bacteria on paper. Appl. Microbiol. Biotechnol. 102, 367375 (2018).

32. Worramongkona, P. et al. A Simple Paper-based Colorimetric Device for Rapid and Sensitive Urinary Oxalate Determinations. Anal. Sci. 34, 103-108 (2018).

33. Asakuma, S. et al. Physiology of consumption of human milk oligosaccharides by infant gut-associated bifidobacteria. J. Biol. Chem. 286, 34583-34592 (2011).

34. Enam, F. \& Mansell, T. J. Analysis of Fucosylated Human Milk Trisaccharides in doi:10.3791/59253

35. Enam, F. \& Mansell, T. J. Linkage-Specific Detection and Metabolism of Human Milk Oligosaccharides in Escherichia coli. Cell Chemical Biology (2018). doi:10.1016/j.chembiol.2018.06.002

36. Katayama, T. et al. Molecular cloning and characterization of Bifidobacterium bifidum 1,2alpha-L-fucosidase (AfcA), a novel inverting glycosidase (glycoside hydrolase family 95$)$. J. Bacteriol. 186, 4885-4893 (2004).

37. Iselt, M., Holtei, W. \& Hilgard, P. The tetrazolium dye assay for rapid in vitro assessment of cytotoxicity. Arzneimittelforschung 39, 747-749 (1989).

38. Choi, H. S., Kim, J. W., Cha, Y.-N. \& Kim, C. A quantitative nitroblue tetrazolium assay for determining intracellular superoxide anion production in phagocytic cells. J. Immunoassay 
Immunochem. 27, 31-44 (2006).

39. Lim, H. H. \& Buttery, J. E. Determination of ethanol in serum by an enzymatic PMS-INT colorimetric method. Clin. Chim. Acta 75, 9-12 (1977).

40. Yang, Y. et al. Paper-Based Microfluidic Devices: Emerging Themes and Applications. Anal. Chem. 89, 71-91 (2017).

41. Martinez, A. W., Phillips, S. T., Butte, M. J. \& Whitesides, G. M. Patterned Paper as a Platform for Inexpensive, Low-Volume, Portable Bioassays. Angew. Chem. Int. Ed Engl.

42. Samyn, P. Wetting and hydrophobic modification of cellulose surfaces for paper applications. J. Mater. Sci. 48, 6455-6498 (2013).

43. Carrilho, E., Phillips, S. T., Vella, S. J., Martinez, A. W. \& Whitesides, G. M. Paper microzone plates. Anal. Chem. 81, 5990-5998 (2009).

44. Carrilho, E., Martinez, A. W. \& Whitesides, G. M. Understanding wax printing: a simple micropatterning process for paper-based microfluidics. Anal. Chem. 81, 7091-7095 (2009). coli strains with chromosomally integrated expression cassettes for the synthesis of 2ltextasciiacutex-fucosyllactose. Microb. Cell Fact. 12, 40 (2013).

46. Huang, D. et al. Metabolic engineering of Escherichia coli for the production of 2'fucosyllactose and 3-fucosyllactose through modular pathway enhancement. Metab. Eng. 41, 23-38 (2017).

47. Albermann, C., Piepersberg, W. \& Wehmeier, U. F. Synthesis of the milk oligosaccharide 2'-fucosyllactose using recombinant bacterial enzymes. Carbohydr. Res. 334, 97-103 (2001).

48. Erney, R. M. et al. Variability of human milk neutral oligosaccharides in a diverse population. J. Pediatr. Gastroenterol. Nutr. 30, 181-192 (2000).

49. McGuire, M. K. et al. What's normal? Oligosaccharide concentrations and profiles in milk 
produced by healthy women vary geographically. Am. J. Clin. Nutr. 105, 1086-1100 (2017).

50. Maltesen, M. J. \& van de Weert, M. Drying methods for protein pharmaceuticals. Drug Discov. Today Technol. 5, e81-8 (2008).

51. Liao, Y.-H., Brown, M. B. \& Martin, G. P. Investigation of the stabilisation of freeze-dried lysozyme and the physical properties of the formulations. Eur. J. Pharm. Biopharm. 58, 1524 (2004).

52. Totten, S. M. et al. Comprehensive profiles of human milk oligosaccharides yield highly sensitive and specific markers for determining secretor status in lactating mothers. $J$. Proteome Res. 11, 6124-6133 (2012).

53. Pausan, M.-R., Kolovetsiou-Kreiner, V., Richter, G. L. \& Madl, T. Human Milk Oligosaccharides modulate the risk for preterm birth in a microbiome dependent and independent manner. doi:10.1101/683714

54. Rudloff, S., Pohlentz, G., Borsch, C., Lentze, M. J. \& Kunz, C. Urinary excretion of in vivo

55. Goehring, K. C., Kennedy, A. D., Prieto, P. A. \& Buck, R. H. Direct evidence for the presence of human milk oligosaccharides in the circulation of breastfed infants. PLoS One

56. Prenner, C., Mach, L., Glössl, J. \& März, L. The antigenicity of the carbohydrate moiety of an insect glycoprotein, honey-bee (Apis mellifera) venom phospholipase A2. The role of

644 57. Rodríguez-Díaz, J., Monedero, V. \& Yebra, M. J. Utilization of natural fucosylated alpha 1,3-fucosylation of the asparagine-bound N-acetylglucosamine. Biochem. J 284 ( Pt oligosaccharides by three novel alpha-L-fucosidases from a probiotic Lactobacillus casei strain. Appl. Environ. Microbiol. 77, 703-705 (2011).

58. Gish, R. G. Early detection of hepatocellular carcinoma through surveillance using biomarkers. Gastroenterol. Hepatol. 10, 121-123 (2014). 
A

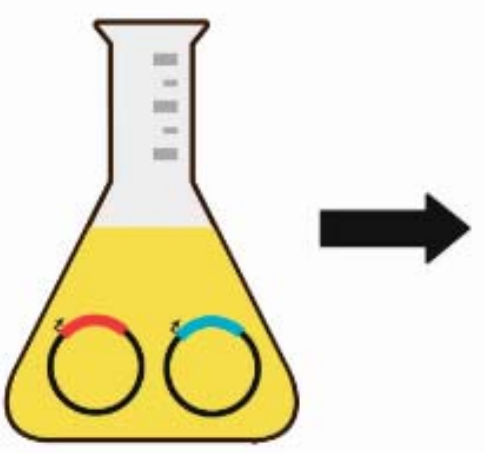

Heterologous expression of enzymes

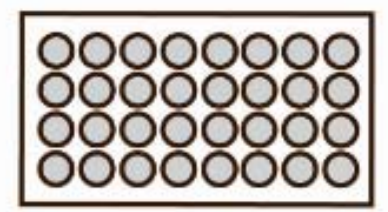

Immobilization of enzymes on paper

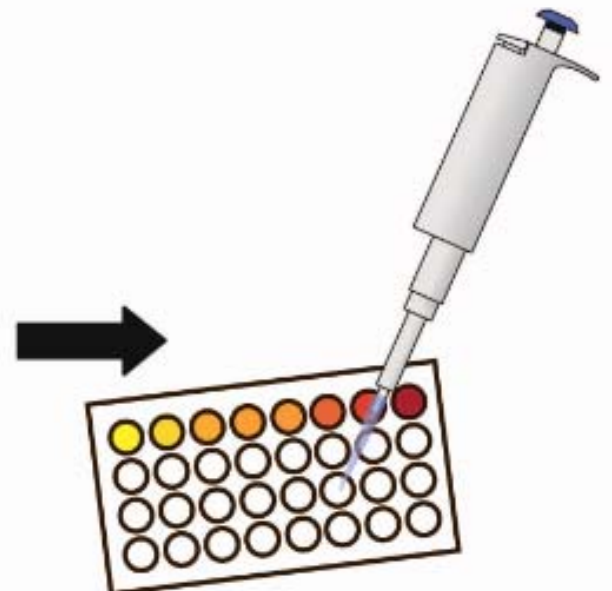

Colorimetric fucosylation assay

B

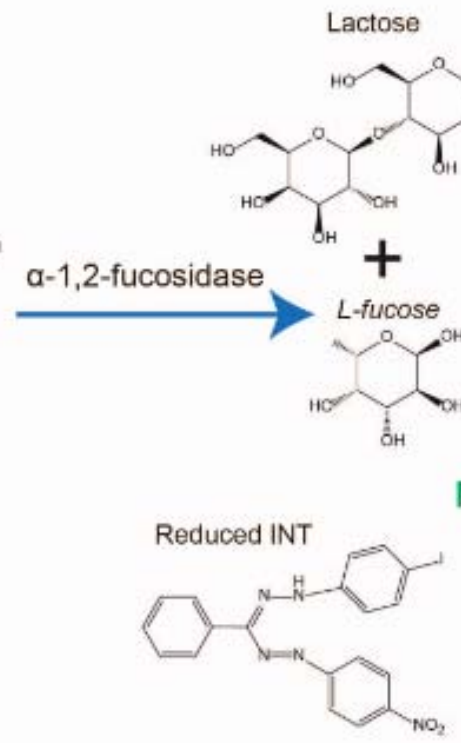

Fucono-1,5-lactone

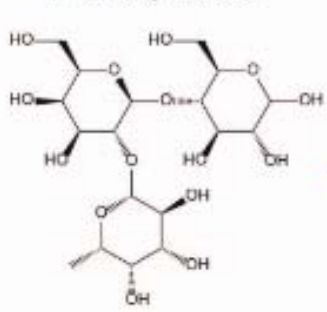

C

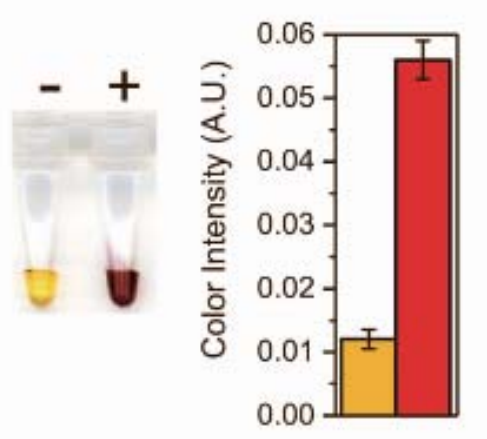

D

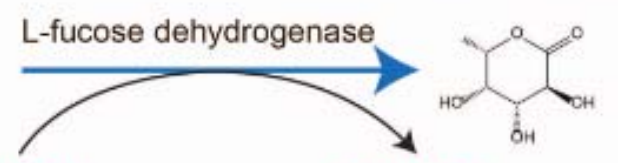

NADP+

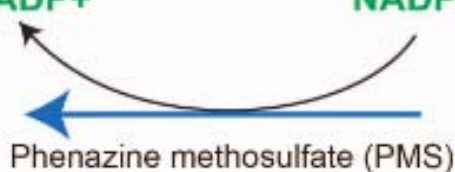

Phenazine methosulfate (PMS)
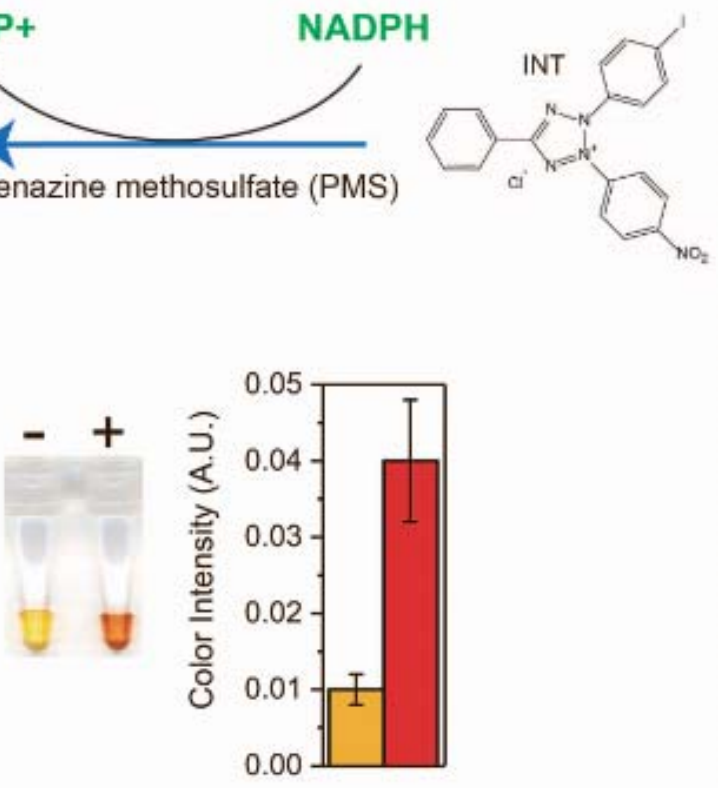

Figure 1: Strategy for paper-based sensing of fucosylation. (A) Schematic illustrating the

651 immobilization of the enzymes on to the paper device and development of colorimetric signal 
653 specific fucosidase coupled to a colorimetric assay based on INT tetrazolium dye, 2'-FL shown

654 here. (C) Images of reaction with free fucose (left) and 2'-FL (right) at $300 \mathrm{mg} / \mathrm{L}$.

A

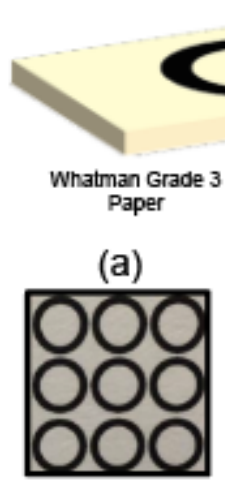

Top (b)

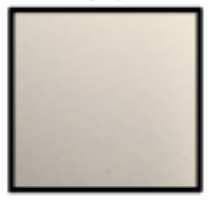

Bottom

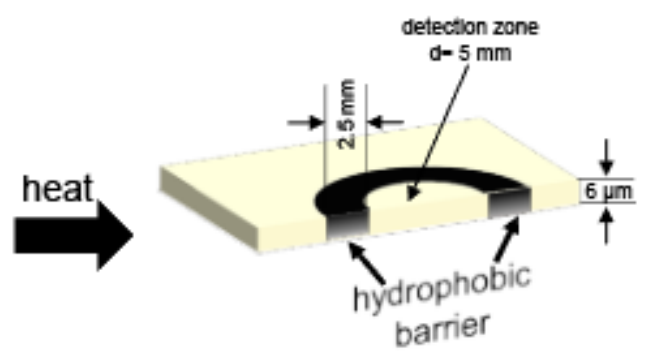

(a)

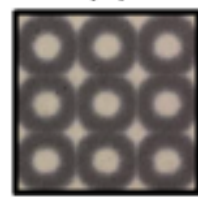

Top (b)

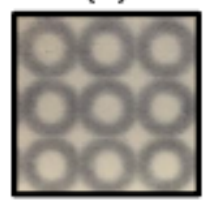

Bottom
B

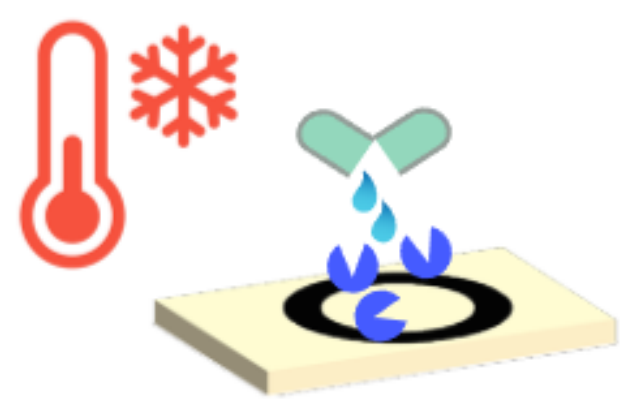

$\mathrm{C}$

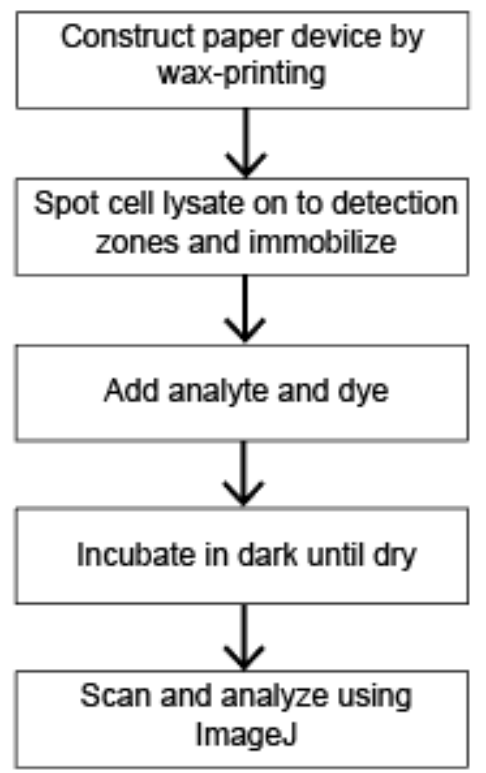
assay. (A) Layout of the wax printed array. Top panel shows the dimensions of the wells. 
660 the wells and immobilized. (C) Overview of the workflow of the assay.

A

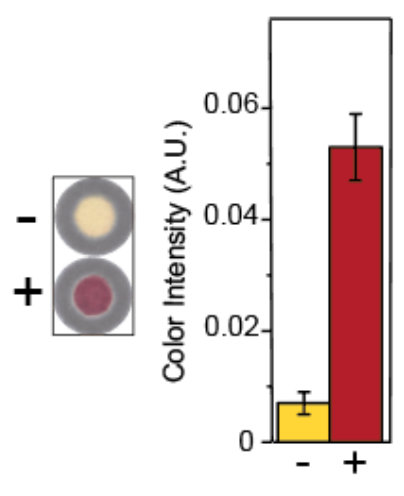

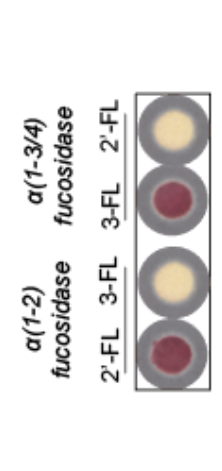

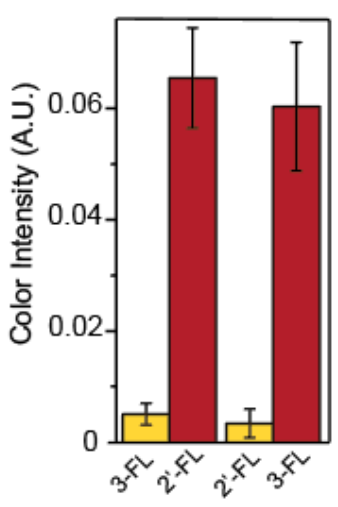

B
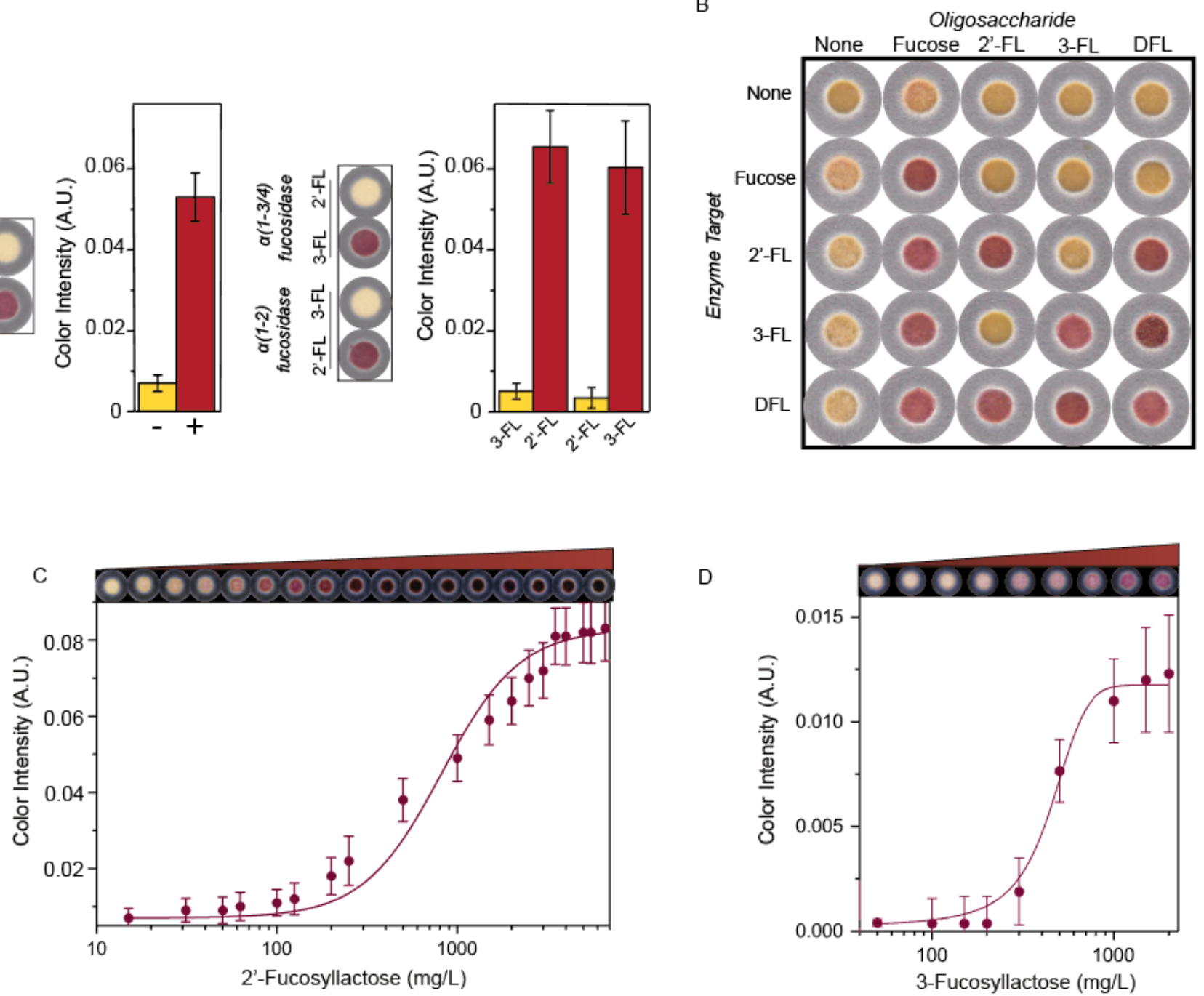

D

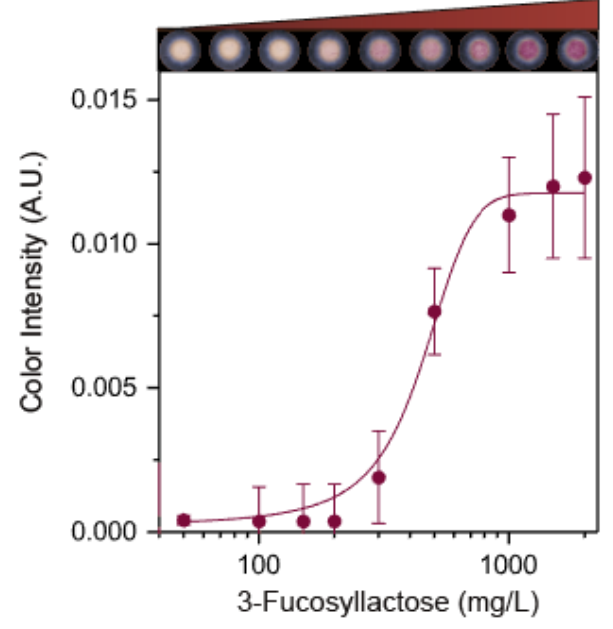


mean color intensity (analyzed in triplicate). The dose-response curve was fitted with a

668 4PL model, shown by the line. The top shows a capture of the signal expression from

669 increasing concentrations of 2'-FL. (D) Calibration curve for 3-FL, as described in (C).

670 Error bars represent standard deviation of triplicate measurements of mean color

671 intensities.

672 


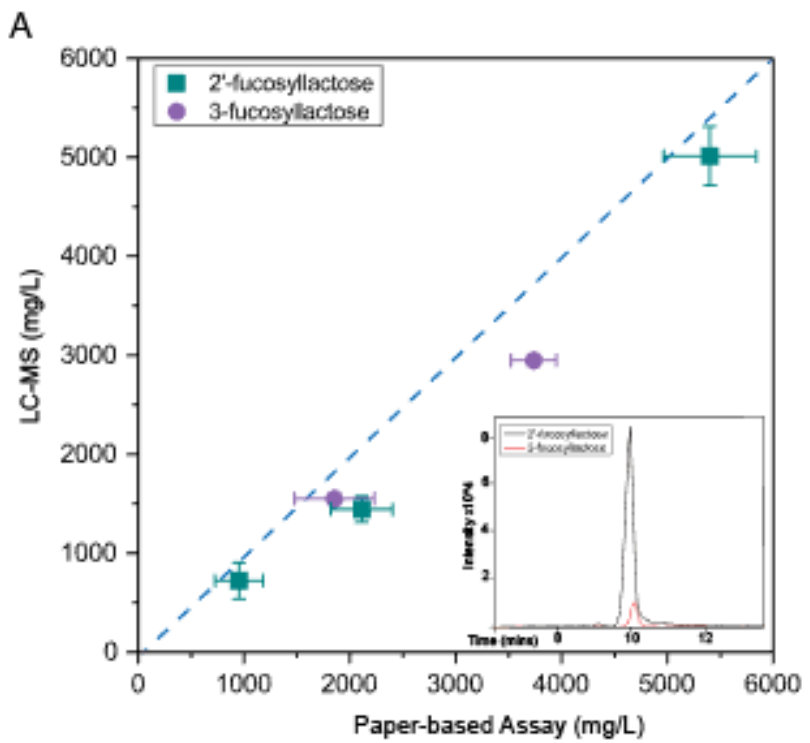

D

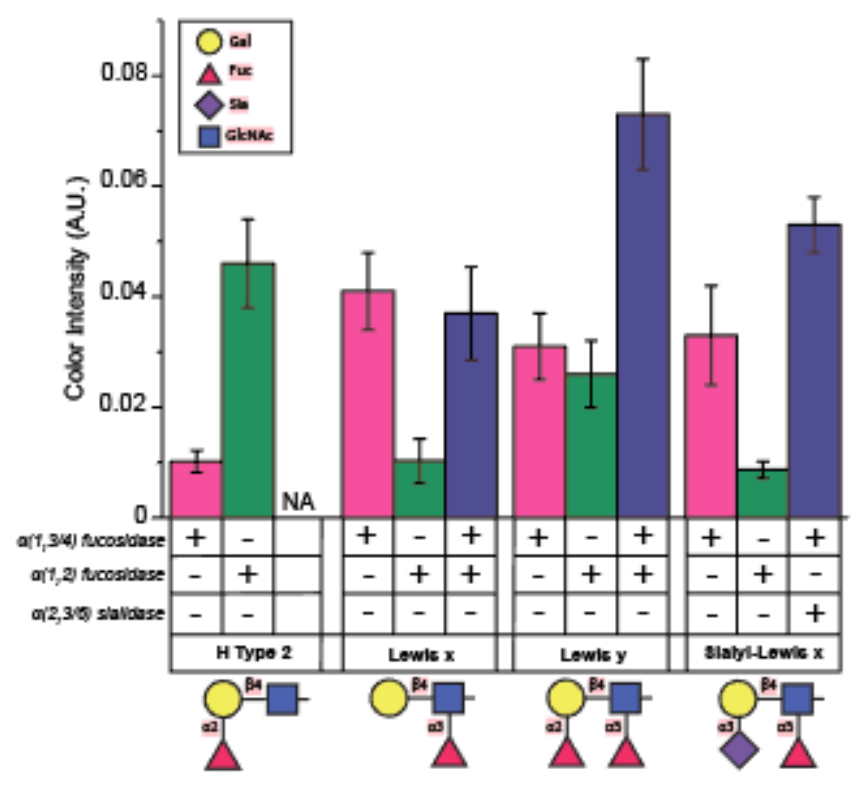

B

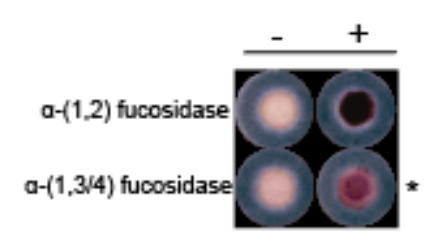

C

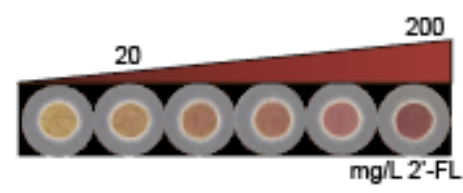

E

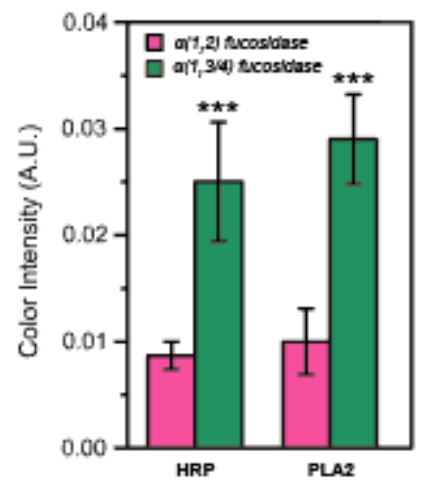

F

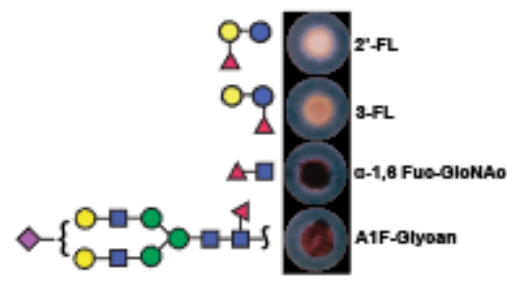

674 Figure 4: Paper-based assay enables determination of fucosylation type in biological

675 fluids and glycoconjugates. (A) Comparing measurement of concentrations of 2'-FL and 3-FL

676 in human breast milk using paper-based assay and HPLC/MS. Lipids and proteins were

677 removed before analysis. To analyze 3-FL, samples were concentrated. Inset shows 
678 chromatogram peaks for 2'-FL (black) and 3-FL (red). (B) Arrayed image showing colorimetric

679 output from assay on breast milk samples. * indicates concentrated samples tested. (C) Testing

680 for 2'-FL in spiked synthetic urine at levels found in maternal and infant urine. (D)

681 Distinguishing Lewis Type 2 antigens and quantification of their fucosylation. (E) a-1,3-

682 Fucosylation in glycoproteins. Differences in the means of the test conditions and the controls

683 were analyzed using a t-test ( $\left.{ }^{* * *} \mathrm{P}<0.0001\right)$. (F) Determination of $\alpha-1,6$-fucosylation in $N$ -

684 glycans. 2'-FL and 3-FL were used as controls. 